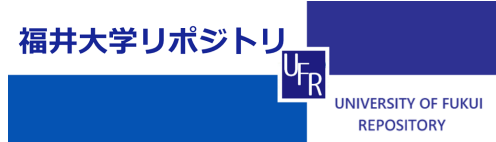

Coh1- APC Control s Axonal Growth and Patterni ng in the Manmal i an $\mathrm{Br}$ ai $\mathrm{n}$

\begin{tabular}{|c|c|}
\hline 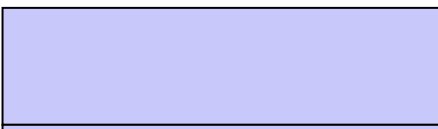 & $\begin{array}{l}\text { KON SH Yoshi yuki, STEGMLLER Judi th, MATSUDA } \\
\text { Takahi ko, BONN Shi ri n, BONN Azad }\end{array}$ \\
\hline $\begin{array}{l}\text { j our nal or } \\
\text { publ i cat i on ti tl e }\end{array}$ & Sci ence \\
\hline vol une & 303 \\
\hline page $r$ ange & $1026-1030$ \\
\hline year & 2004 \\
\hline URL & ht t p: //hdl . handl e. net /10098/5230 \\
\hline
\end{tabular}




\section{Cdh1-APC Controls Axonal Growth and Patterning in the Mammalian Brain}

\author{
Yoshiyuki Konishi, ${ }^{1}$ Judith Stegmüller, ${ }^{1}$ Takahiko Matsuda, ${ }^{2}$ \\ Shirin Bonni, ${ }^{3}$ Azad Bonni ${ }^{1}$ *
}

The anaphase-promoting complex (APC) is highly expressed in postmitotic neurons, but its function in the nervous system was previously unknown. We report that the inhibition of Cdh1-APC in primary neurons specifically enhanced axonal growth. Cdh1 knockdown in cerebellar slice overlay assays and in the developing rat cerebellum in vivo revealed cell-autonomous abnormalities in layer-specific growth of granule neuron axons and parallel fiber patterning. Cdh1 RNA interference in neurons was also found to override the inhibitory influence of myelin on axonal growth. Thus, Cdh1-APC appears to play a role in regulating axonal growth and patterning in the developing brain that may also limit the growth of injured axons in the adult brain.

The ubiquitin ligase anaphase-promoting complex (APC) is essential for coordination of cell cycle transitions, including mitotic exit (1-3). APC activity is stimulated by the regulatory proteins $\mathrm{Cdc} 20$ and $\mathrm{Cdh} 1$ in a cell cycle-dependent manner. Cdc20 association with APC is required for APC activity during early mitosis, but Cdh1 association is required for APC activity during late mitosis and G1 $(4,5)$. Cdh1 and core components of APC are also expressed in post-

${ }^{1}$ Department of Pathology, ${ }^{2}$ Department of Genetics, Harvard Medical School, 77 Avenue Louis Pasteur, Boston, MA 02115, USA. ${ }^{3}$ Department of Biochemistry and Molecular Biology, University of Calgary, 3330 Hospital Drive Northwest, Calgary, Alberta T2N 4N1, Canada.

*To whom correspondence should be addressed. Email: azad_bonni@hms.harvard.edu mitotic neurons in the mammalian brain $(6,7)$.

To characterize Cdh1 function in postmitotic neurons, we used a DNA template-based RNA interference (RNAi) method to acutely knock down the expression of Cdh1 in primary cerebellar granule neurons (8). The expression of cdh1 small hairpin RNAs (shedh1) reduced Cdh1 expression in COS cells and in primary neurons (fig. S1, A and B). The shcdh1-expressing granule neurons did not reenter the cell cycle, failing to incorporate bromodeoxyuridine or undergo cytokinesis (9). Cdh1 knockdown had no effect on the survival of the granule neurons (fig. S1C). However, Cdh1 knockdown altered the morphology of neuronal processes, raising the possibility that $\mathrm{Cdh} 1$ might regulate neuronal morphogenesis.

To determine the role of Cdh1 in neuronal morphogenesis, we transfected primary cere- bellar granule neurons from postnatal day 6 (P6) rat pups with the control U6 or U6/shcdh1 plasmid at the time of plating, when these neurons just begin to grow axons (8). Each day for 6 days after transfection, we measured the length of axons and dendrites in the same transfected granule neurons. Axons and dendrites of transfected neurons were identified on the basis of their morphology and by the expression of the axonal marker Tau and the somato-dendritic marker MAP2 (Fig. 1A). While the expression of shcdh1 in granule neurons had little effect on the generation and growth of dendrites, Cdh1 knockdown led to a dramatic increase in axonal length (Fig. 1, B and C), both accelerating the rate of growth and significantly augmenting the final axonal length (Fig. 1C). The vast majority of transfected cells expressed the neuronspecific $\beta$-tubulin type III (Tuj1), a marker of postmitotic granule neurons that was not affected by Cdh1 RNAi (Fig. 1E). Thus, Cdh1 specifically inhibits axonal but not dendritic growth in postmitotic granule neurons.

Cdh1 RNAi lowered the numbers of neurons with short axons and increased the numbers of neurons with long axons, which supports the idea that Cdh1 knockdown did not operate in a subpopulation of neurons (Fig. 1D). In other experiments, Cdh1 knockdown increased the total length but not the number of branches of granule neuron axons (9), which suggests that Cdh1 knockdown specifically enhances the growth of the major axonal fibers in neurons. A second construct encoding small hairpin cdh1 RNAs (shcdh1-b) that reduced the expression of Cdh1 also promoted the growth of axons (Fig. 1F). shRNAs to several other genes encoding proteins unrelated to Cdh1, including the transcription factor MEF2A (10), failed to promote axonal growth (Fig. 1F). 
In proliferating cells, Cdh1 forms a complex with APC and mediates its effects on the cell cycle through the ubiquitin ligase activity of $\operatorname{APC}(4,5,11)$. We found that Cdh1 forms a complex with APC in neurons; this complex is predominantly localized in the nucleus (Fig. 2, A and B). Whole-cell or nuclear lysates from granule neurons contained high APC ubiquitin ligase activity that was immunoprecipitated from granule neuron lysates with an antibody to $\mathrm{Cdc} 27$, a component of APC (Fig. 2C) (9). Thus, Cdh1 interacts constitutively with APC in the nucleus of postmitotic neurons and endows neurons with robust APC ubiquitin ligase activity.

We generated a dominant interfering form of the APC E3 component APC11 by a point mutation of the APC11 RING finger motif (APC11C73A) $(12,13)$. Upon expression in granule neurons, APC11C73A, but not wildtype APC11, significantly induced axonal growth (Fig. 2D). The APC inhibitor protein Emil interacts with APC through its Cterminal zinc-binding region (ZBR) $(14,15)$. The expression of full-length Emi1, but not an Emil protein lacking the C-terminal ZBR (Emi1 $\Delta$ ZBR), in proliferating cells increased the fraction of cells in $S$ phase and $G 2 / M$, consistent with Emil's ability to inhibit APC activity (Fig. 2E) (16). In axon growth assays in granule neurons, expression of Emil, but not Emi1 $\Delta$ ZBR, significantly increased the growth of axons (Fig. 2E). Thus, our results suggest that the ubiquitin ligase activity of endogenous Cdh1-APC complex suppresses axonal growth in neurons.

Having identified a role for endogenous Cdh1 and APC activity in the control of axonal growth in primary postmitotic granule neurons, we next characterized the function of Cdh1-APC in the context of the intact cerebellar cortex. To do this, we devised a slice overlay assay for the developing cerebellum (8). We plated P6 granule neurons, in which Cdh1 RNAi was triggered, on top of organotypic cerebellar slices from P9 rat pups (Fig. 3A). Seventy-two hours later, the cerebellar slices were fixed and subjected to immunohistochemistry. Granule neurons in which Cdh1 RNAi was triggered exhibited longer axons than the control U6-transfected granule neurons (Fig. 3, B and C). Examination of the transfected granule neurons in the cerebellar slice overlay assays also revealed a significant difference in the spatial preference of granule neuron axonal growth on the cerebellar tissue (Fig. 3, B and C). About $60 \%$ of U6-transfected granule neurons extended axons along the molecular and external granule layers. By contrast, only $29 \%$ of shcdh1expressing granule neurons grew their axons along these layers. About $56 \%$ of the shcdh1expressing granule neurons extended axons in random directions that crossed the distinct layers of the cerebellar cortex. More than half of these shcdh1-expressing neurons elaborated long axons over the cerebellar white matter. Less than a third of control U6transfected granule neurons grew axons in random directions, and a small fraction of these neurons extended long axons over the white matter. Thus, beyond inhibiting axonal elongation per se, Cdh1 cell autonomously limits the growth of axons in a layer-specific manner in the context of the developing cerebellar cortex. In addition, the distinct responses of control and shcdh1-expressing granule neuron axon growth over white mat-
A
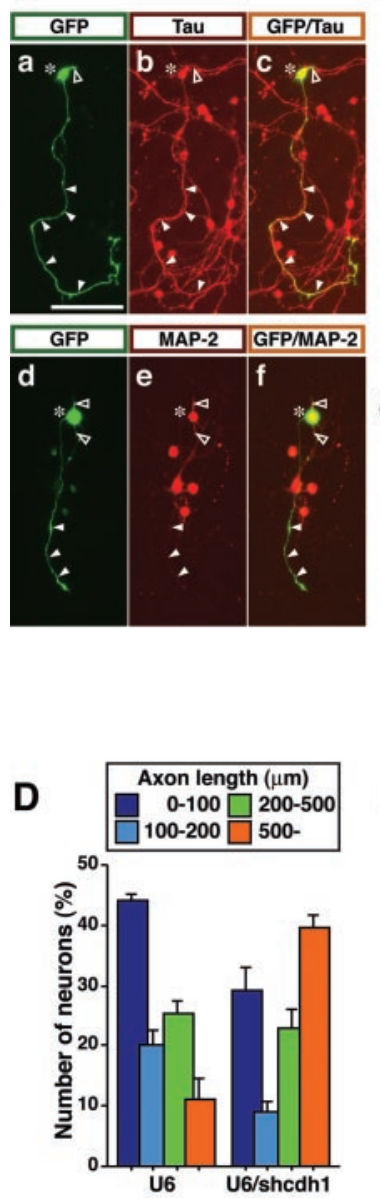

B

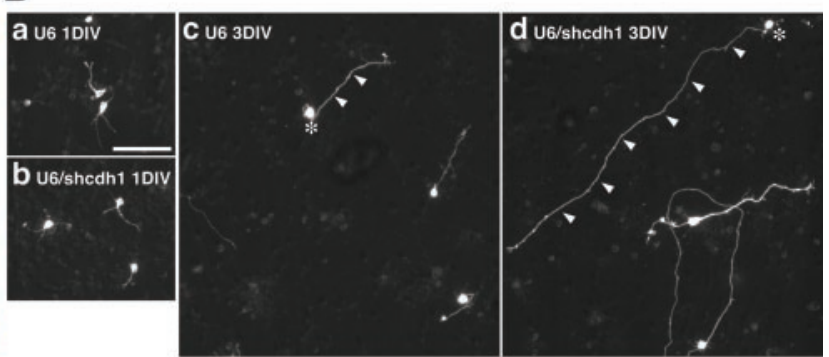

C
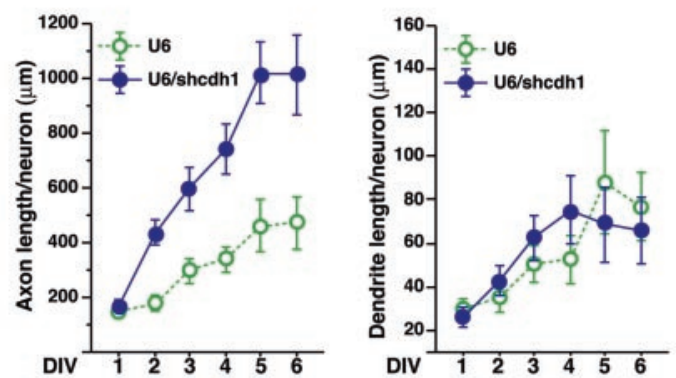

E

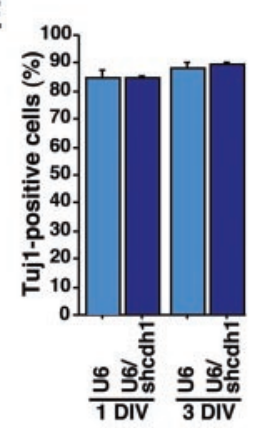

F
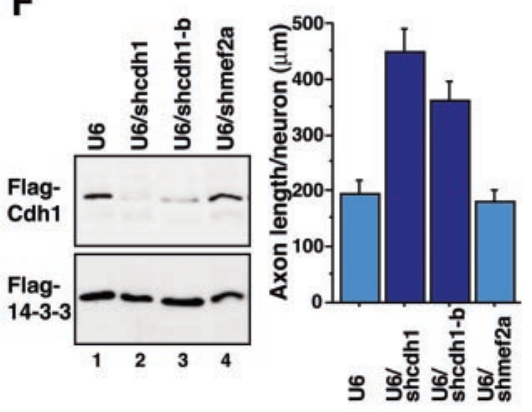

Fig. 1. Cdh1 knockdown triggers marked and specific enhancement of axonal growth in postmitotic neurons. (A) Cerebellar granule neurons were transfected 8 hours after plating with the U6 control plasmid, together with expression plasmids encoding GFP and $\mathrm{Bcl}-\mathrm{xl}(8)$. Neurons were then placed in serum-free medium containing insulin, and 3 days later were subjected to immunocytochemistry with an antibody to GFP and an antibody to Tau or MAP2 (Sigma). Asterisks, open arrowheads, and closed arrowheads, respectively, indicate the cell body, dendrites, and axons. (B and C) Cerebellar granule neurons were transfected with the control U6 or U6/shcdh1 plasmid, together with the GFP and $\mathrm{BCl}-\mathrm{xl}$ expression plasmids, and analyzed as in (A) at 1 to 6 days in vitro culture (DIV). Total axonal and dendritic length were measured in the control U6-transfected or U6/shcdh1-expressing neurons (8). Cdh1 knockdown significantly increased axonal but not dendritic length (ANOVA, $P<$ 0.005 at 3 to 6 DIV). A total of more than 600 neurons were analyzed for each construct. (D) Neurons were transfected with the $\mathrm{U} 6$ or $\mathrm{U} 6 / \mathrm{shcdh} 1$ plasmid as in (A), analyzed at 3 DIV, and classified on the basis of axon length. The majority of control U6-transfected neurons had short axons, whereas the population of neurons with long axons was markedly increased upon Cdh1 RNAi. Values represent mean $+/-$ SEM number of neurons (\%) with indicated axonal length in three independent experiments. More than 150 neurons were measured per construct. (E) Granule neurons were transfected as in (B) and (C), fixed at 1 or 3 DIV, and subjected to immunocytochemistry with the GFP antibody and the Tuj1 antibody (Covance). (F) Left panel: Lysates of COS cells that were transfected with the control U6 or indicated U6/shRNA plasmid, together with expression plasmids encoding Flag-14-3-3 and Flag-Cdh1, were immunoblotted with an antibody to Flag (Sigma). Right panel: Granule neurons were transfected with the control U6, U6/shcdh1, U6/shcdh1-b, or U6/shmef2a plasmid. After 3 days, axonal length was measured as in (C). Expression of shcdh1 and shcdh1-b but not shmef2a resulted in significant increase in axon length when compared with control U6-transfected granule neurons (ANOVA, $P<0.0001$ ). More than 150 cells were analyzed in each condition in three independent experiments. 
Fig. 2. Cdh1 resides in the nucleus in postmitotic neurons and forms a complex with APC whose ubiquitin ligase activity suppresses axonal growth. (A) Nuclear (NE) and postnuclear supernatant (PNS) fractions prepared from cerebellar granule neurons (8) were subjected to immunoblotting using antibodies to $\mathrm{Cdh} 1$ (Pharmingen) and Cdc27 (Santa Cruz). Cdh1 and $\mathrm{Cdc} 27$ were predominantly expressed in the nuclear fraction, together with the APC core subunit Cdc27. (B) Whole-cell lysates from cerebellar granule neurons (3 DIV) were precipitated with beads that were conjugated with the $\mathrm{Cdc} 27$ antibody and subjected to immunoblotting with the Cdh1 antibody. (C) Endogenous APC from cerebellar granule neurons or HeLa cells were immunoprecipitated with the Cdc27 antibody (8). (D) Left panel: Lysates of 293T cells that were transfected with an expression plasmid encoding Flag-tagged wildtype APC11 (APC11WT) or the mutant form of APC11 (APC11C73A) were immunoblotted with the Flag antibody. Right panel: Granule neurons were transfected with the Flag-tagged APC11WT or APC11C73A expression plasmid or the control plasmid. Axon length of transfected neurons was measured as in Fig. 1C. APC11C73A but not APC11WT significantly increased the axon length (ANOVA, $P<0.0001$ ). More than 150 neurons were measured for each condition in three independent experiments). (E) Left panel: Lysates of 293T cells that were transfected with an expression plasmid encoding Flag-tagged wild-type Emi1 (Emi1WT), a mutant Emi1 that lacks zinc-binding region (Emi1 $\triangle$ ZBR), or the control vector were immunoblotted with the Flag antibody. Middle panel: The 293T cells that were transfected with the Flag-tagged Emi1WT, Emi1 $\Delta$ ZBR, or their control vector, together with the GFP expression plasmid. Two days after transfection, cells were subjected to fluorescence-activated cell sorting analysis, and the DNA
B

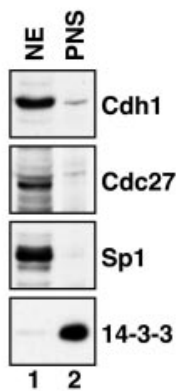

D

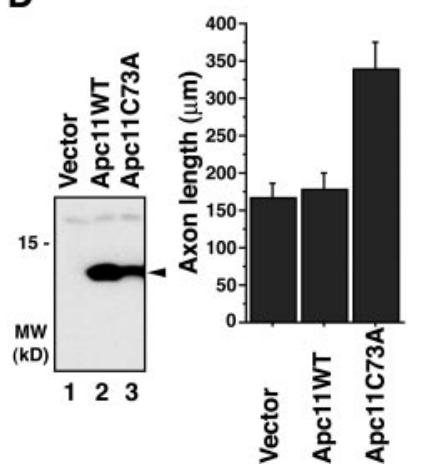

C

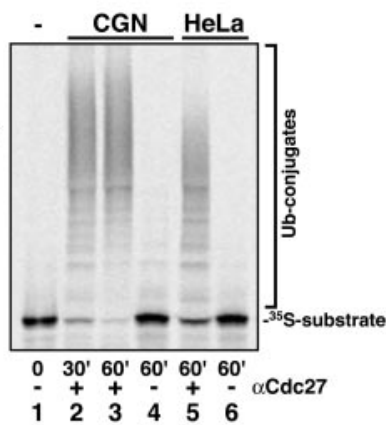

$\mathbf{E}$

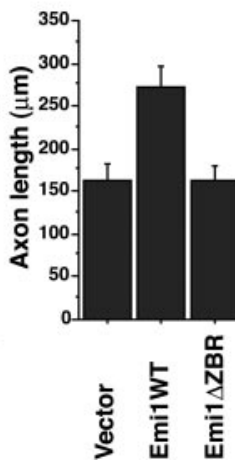

content within the GFP-positive population of cells was analyzed ( $x$ axis, DNA content; $y$ axis, number of cells). Right panel: Axon length of control vector-transfected, Emi1WT-, or Emi1 $\Delta$ ZBR-expressing neurons was analyzed as in Fig. 1C. Emi1WT, but not Emi $1 \Delta Z B R$, increased axon length in granule neurons when compared with control vector (ANOVA, $P<$ $0.0001)$. More than 150 neurons were measured for each condition in three independent experiments.

Fig. 3. Cdh1 RNAi disrupts the layer-specific growth of granule neuron axons in cerebellar slice overlay assays. (A) Schematic representation of cerebellar slice overlay assays (8). Granule neurons from P6 rat pups were harvested and transfected in suspension with the U6 or U6/shcdh 1 plasmid that also encoded GFP. These granule neurons were then plated on top of P9 cerebellar slices. Slices were analyzed 72 hours later with immunohistochemistry by using the GFP antibody to label transfected neurons, an antibody to Calbindin (Cal) to label Purkinje neurons, and Hoechst to reveal the anatomical organization of the cerebellar cortex. (B) The majority of control U6-transfected granule neurons extended axons characteristically along the molecular layer and EGL or toward these layers. By contrast, the shcdh1-expressing neurons extended axons that did not respect the layer boundaries, and many of these axons grew over the white matter. Calibration bar, $300 \mu \mathrm{m}$. EGL, external granule layer; ML, molecular layer; PL, Purkinje cell layer; GCL, granule cell layer; WM, white matter. (C) Results in Fig. 3B were quantitated in a blinded manner. Left panel: Axonal length was significantly higher in U6/shcdh1-expressing neurons ( $t$ test, $P<0.05$ ). The total number of neurons measured was 200 in two independent experiments). Table and right panel: U6-transfected granule neuron axons most commonly extended along the EGL and molecular layers, whereas axons in shcdh1-expressing neurons extended in random directions.
A

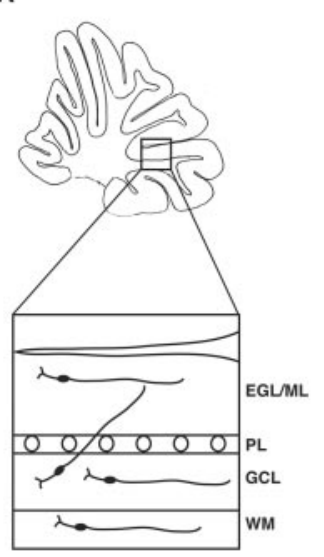

B

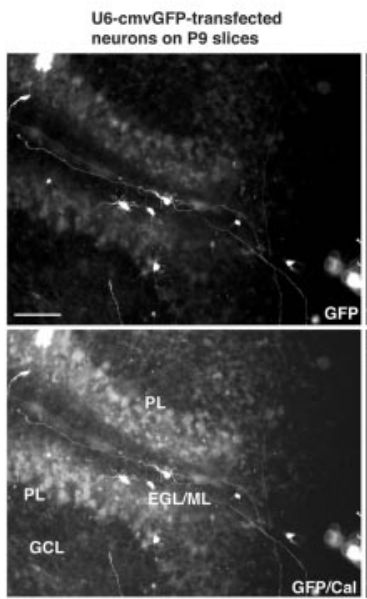

C

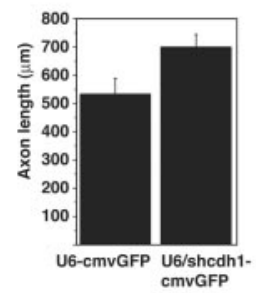

Direction of axonal growt On EGL/ML From other layers towards EGLML Random direction cmvGFP
U6-cmvGFP U6/shedh1-cmvGFP

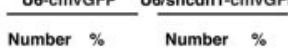

$\begin{array}{llll}130 & 60.2 & 67 & 28.6\end{array}$

$27 \quad 12.5 \quad 36$

$\begin{array}{llll}59 & 27.3 & 131 & 56\end{array}$
U6/shedh1-cmvGFP-transfected neurons on $\mathrm{P} 9$ slices
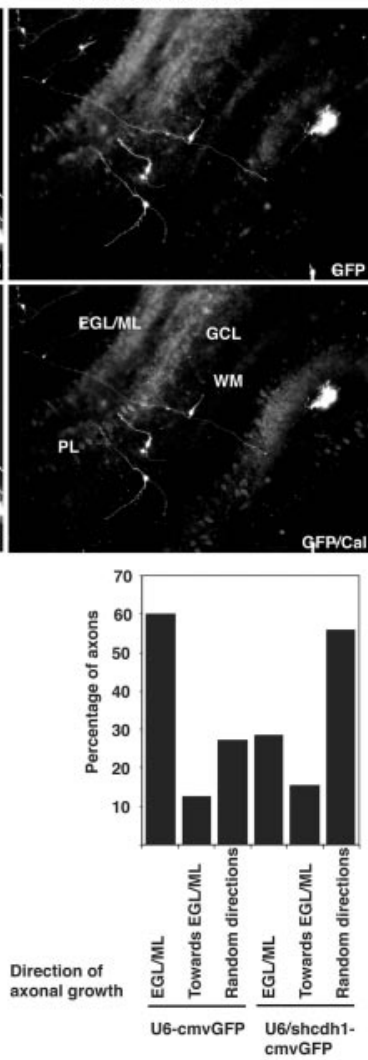

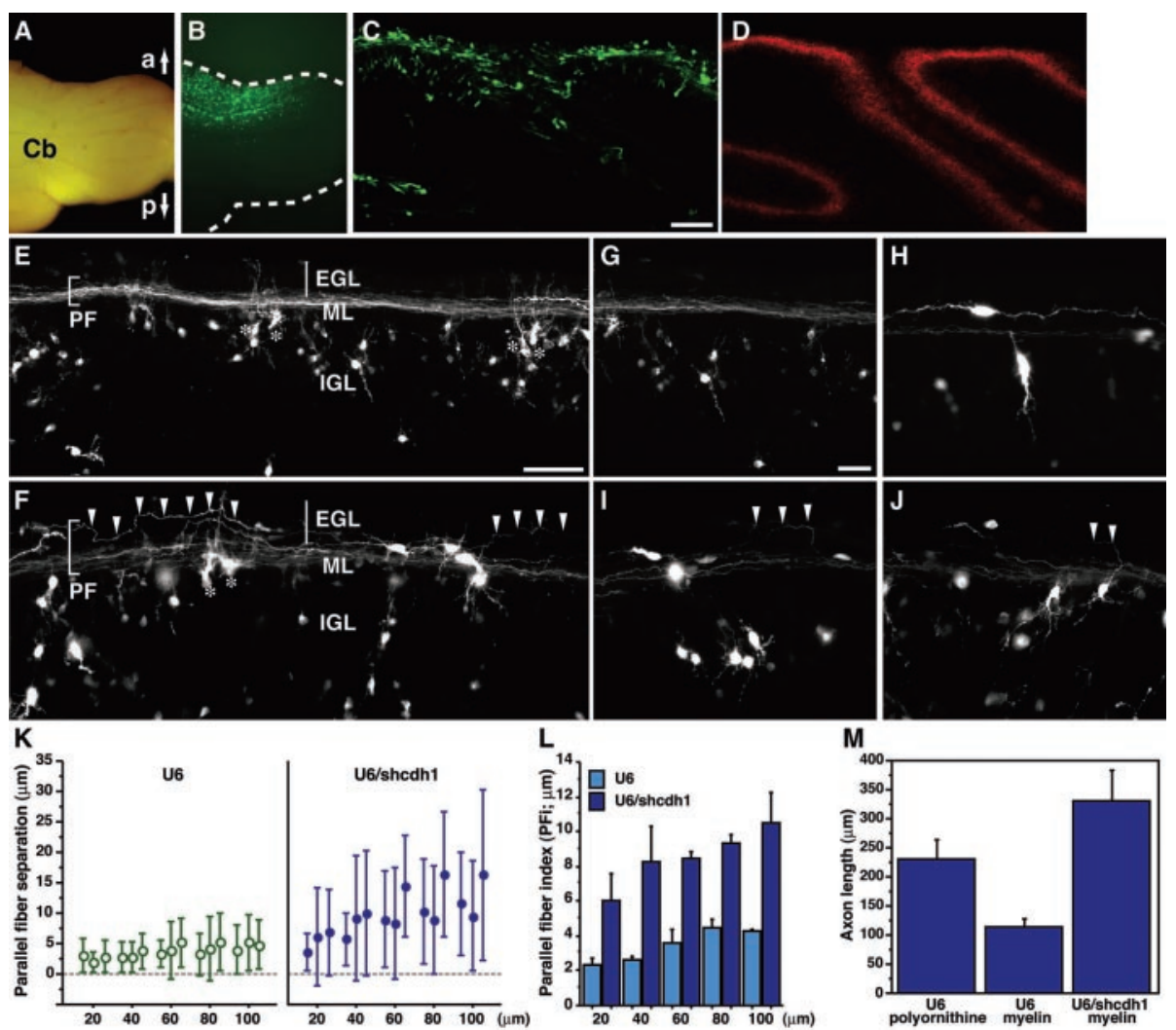

M

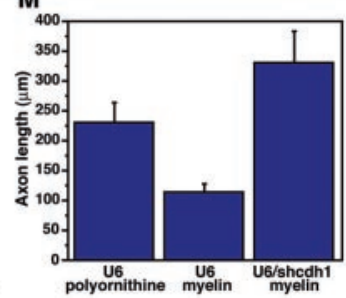

Fig. 4. Cdh1 knockdown disrupts axonal patterning in the mammalian brain in vivo and overrides myelin inhibition of axonal growth. (A and B) P3 rat pups were injected and electroporated with expression plasmids encoding GFP and $\mathrm{Bcl}-\mathrm{xl}$ into the cerebellum (8). Three days later, the cerebellum was dissected and transfected cells were visualized [(A), bright field; (B), GFP)]. a, anterior; P, posterior. (C and D) Sections of GFP-electroporated cerebellum were prepared and subjected to immunofluorescence with the GFP antibody (C) and an antibody to the EGL neuron marker TAG1 (D). (E to J) Postnatal day 3 rat pups were electroporated as in (A) and (B), together with the $\mathrm{U} 6$ control $[(E),(G)$, and $(\mathrm{H})]$ or the U6/shcdh1 plasmid $[(\mathrm{F}),(\mathrm{I})$, and $(\mathrm{J})]$. Five days later, paralobular sections were prepared and subjected to immunohistochemistry with the GFP antibody. In control cerebellum, granule neuron axons formed neatly stacked parallel fibers (PF) in the molecular layer (ML). By contrast, Cdh1 knockdown resulted in disorganization of parallel fibers (arrowheads). Asterisks indicate non-neuronal cells. (K) Pairs of parallel fibers were randomly selected and the separation between the two fibers was measured as described (8). Distance from reference point ( $x$ axis) and difference in the separation of the pairs of parallel fibers ( $y$ axis) were measured in three different animals (mean $+/-$ SD). A total of 60 pairs of parallel fibers were analyzed in each condition. U6/shcdh1-expressing neurons showed greater change in the separation between pairs of parallel fibers. (L) The parallel fiber index (PFi) was calculated at each point along the $x$ axis as described (8). In control animals, axons of cerebellar granule neurons were closer to each other and thus had a low PFi. In contrast, U6/shcdh1-transfected animals show significantly higher PFi (ANOVA, $P<0.005$ at 40 to $100 \mu \mathrm{m}$ ), which suggests that parallel fibers are disorganized and have greater separation between them upon Cdh1 RNAi. (M) P6 granule neurons were transfected with the U6 or U6/shcdh1 plasmid, together with the GFP expression plasmid, placed on polyornithine substrate in the presence or absence of myelin, and analyzed 3 days after transfection (8) (fig. S3). Myelin reduced the growth of U6-transfected axons when compared with polyornithine (ANOVA, $P<0.01$ ), whereas Cdh1 RNAi opposed myelin inhibition and restored axonal growth (as compared with U6 on myelin, ANOVA, $P<0.0001$ ). The total number of neurons analyzed was 103.

ter raised the possibility that Cdh1 knockdown might overcome myelin inhibition of axonal growth.

To establish the role of Cdh1 in the developing cerebellum in vivo, we induced Cdh1 RNAi in the cerebellum of P6 rat pups by electroporation (17). We adapted a method of electroporation (18) so as to trigger Cdh1 RNAi in granule neurons within the developing postnatal rat cerebellum (8). We injected an expression plasmid encoding green fluorescent protein (GFP) into the cerebellar cortex of $\mathrm{P} 3$ rat pups and subjected these pups to electroporation (Fig. 4, A and B). Three days after electroporation, the cerebellum was isolated and subjected to immunohistochemistry. The majority of the GFP-positive cells were located in the TAG1-positive external granule layer (EGL), which suggests that many granule neurons were transfected (Fig. 4, C and D). Some of the GFP-positive granule neurons were located in the internal granule layer (IGL).

We next injected the U6 or U6/shcdh1 plasmid together with the GFP expression plasmid into the cerebellar cortex of P3 rat pups and analyzed these animals 5 days after electroporation. Coronal sections of the cerebellum were prepared to allow the assessment of granule neuron parallel fiber axons. In control U6electroporated animals, GFP-positive granule neurons were found in the EGL and IGL (Fig. 4, E, G, and H). The U6-transfected granule neurons extended neatly stacked parallel fibers located just below the EGL (Fig. 4, E, G, and $\mathrm{H})$. Thus, the characteristics of the control U6transfected granule neurons matched closely the behavior of normally developing granule neurons. The shcdh1-expressing granule neurons were also present in the EGL and IGL (Fig. 4, F, I, and J). The shcdh1-expressing granule neurons extended normal dendrites when compared to the U6-transfected granule neurons (Fig. 4, G to J). By contrast, axons of the shcdh1-expressing granule neurons exhibited striking abnormalities. The parallel fibers of granule neurons in which Cdh1 RNAi was triggered appeared defasciculated (Fig. 4F). In addition, several of these axons wandered off the main track of the parallel fibers and grew abnormally toward the EGL (Fig. 4, F, I, and J).

The in vivo parallel fiber phenotype was quantitated by a blinded observer by measuring the separation between pairs of randomly selected GFP-positive parallel fiber axons in the orthogonal plane to the parallel fibers (Fig. 4K) (8). From these measurements, we calculated an index of parallel fiber defasciculation (PFi) (8), which was significantly greater in shcdh1-expressing neurons than in control U6-transfected neurons (Fig. 4L). These results suggest a physiological, cellautonomous function for $\mathrm{Cdh} 1$ in the control of granule neuron axonal growth and patterning in the developing cerebellum in vivo.

Having established a role for Cdh1 in the control of axonal growth and patterning in the developing mammalian brain, we next turned to the observation in the slice overlay assay (Fig. $3 \mathrm{~B})$, suggesting the possibility that Cdh1 activity might also contribute to the intrinsic inability of mammalian brain neurons to grow when exposed to myelin. We tested the ability of control U6- or U6/shcdh1-transfected cerebellar granule neurons to grow axons over myelin substrate that was prepared from adult rat brain (8). Axonal growth in control-transfected granule neurons was significantly inhibited on myelin as compared with polyornithine substrate (Fig. 4M; figs. S3 and S4). By contrast, the knockdown of $\mathrm{Cdh} 1$ in granule neurons allowed granule neurons to override myelin inhibition of axonal growth, including in a modified stripe assay (Fig. 4M; figs. S3 and S4). Exposure to myelin did not induce the already high level of ubiquitin ligase activity of endoge- 


\section{REPOR T S}

nous APC in granule neurons (9). This result suggests that Cdh1-APC represents a cellintrinsic mechanism that inhibits axonal growth and the knockdown of Cdh1-APC activity overcomes the extrinsic negative influence of myelin on axonal growth

We have described a function for Cdh1APC outside the cell cycle in postmitotic neurons. The inhibition of endogenous APC activity in neurons triggered a robust and specific elongation of axons but not dendrites. In addition to regulating axonal growth, Cdh1 potently controlled axonal patterning and thereby limited granule neuron axonal growth to the molecular layer of the cerebellar cortex. Cdh1 knockdown was also found to override the negative influence of adult rat myelin on axonal growth, even though myelin did not increase the already high level of endogenous APC activity in neurons. Thus, Cdh1-APC represents a cellautonomous regulator of axonal growth and patterning during brain development that may also contribute to the cell-intrinsic inability of injured neurons to extend axons in the adult brain.

In proliferating cells, Cdh1-APC localization in the nucleus appears to be critical for regulation of the cell cycle (19). The majority of Cdh1-APC in postmitotic neurons resides in the nucleus. Ubiquitin-mediated proteasomal protein degradation acting locally within axon terminals is thought to regulate chemo- tropic responses of axon growth cones and axon terminal pruning $(20,21)$. Our results raise the possibility that Cdh1-APC operates in the nucleus to control axonal growth and patterning. Growing evidence suggests that neuron-enriched transcription factors control programs of gene expression dedicated to axonogenesis (22-24). By acting in the nucleus, Cdh1-APC may play a pivotal role in axonal morphogenesis.

\section{References and Notes}

1. J. W. Harper, J. L. Burton, M. J. Solomon, Genes Dev. 16, 2179 (2002).

2. D. O. Morgan, Nature Cell Biol. 1, E47 (1999).

3. W. Zachariae, K. Nasmyth, Genes Dev. 13, 2039 (1999).

4. R. Visintin, S. Prinz, A. Amon, Science 278, 460 (1997).

5. M. Schwab, A. S. Lutum, W. Seufert, Cell 90, 683 (1997).

6. C. Gieffers, B. H. Peters, E. R. Kramer, C. G. Dotti, J. M. Peters, Proc. Natl. Acad. Sci. U.S.A. 96, 11317 (1999).

7. J. M. Peters, Mol. Cell 9, 931 (2002).

8. Materials and methods are available as supporting material on Science Online.

9. Y. Konishi, J. Stegmüller, A. Bonni, unpublished observations.

10. B. Gaudilliere, Y. Shi, A. Bonni, J. Biol. Chem. 277, 46442 (2002)

11. R. W. King et al., Cell 81, 279 (1995).

12. J. D. Leverson et al., Mol. Biol. Cell 11, 2315 (2000).

13. Z. Tang et al., Mol. Biol. Cell 12, 3839 (2001).

14. J. D. Reimann et al., Cell 105, 645 (2001).

15. J. D. Reimann, B. E. Gardner, F. Margottin-Goguet, P. K. Jackson, Genes Dev. 15, 3278 (2001).

16. J. Y. Hsu, J. D. Reimann, C. S. Sorensen, J. Lukas, P. K. Jackson, Nature Cell Biol. 4, 358 (2002).
17. M. Swartz, J. Eberhart, G. S. Mastick, C. E. Krull, Dev. Biol. 233, 13 (2001).

18. T. Matsuda, C. L. Cepko, Proc. Natl. Acad. Sci. U.S.A. 101, 16 (2004).

19. M. Jaquenoud, F. van Drogen, M. Peter, EMBO J. 21, 6515 (2002).

20. D. S. Campbell, C. E. Holt, Neuron 32, 1013 (2001).

21. R. J. Watts, E. D. Hoopfer, L. Luo, Neuron 38, 871 (2003).

22. A. Kania, R. L. Johnson, T. M. Jessell, Cell 102, 161 (2000).

23. R. Shirasaki, S. L. Pfaff, Annu. Rev. Neurosci. 25, 251 (2002).

24. J. M. Weimann et al., Neuron 24, 819 (Dec, 1999).

25. We thank C. L. Cepko for sharing in vivo electroporation technology before publication and for critical reading of the manuscript, W. Harper for critical reading of the manuscript, B. Gaudillière and E. Cooper for helpful discussions, R. King for providing the APC11 plasmid, M. Pagano for providing the Emi1 plasmid, and S. DiBacco and S. Vasquez for technical assistance. Supported by an NIH grant to A.B. (RO1NS41021). J.S. is supported by a fellowship from the Deutsche Forschungsgemeinschaft. A.B. is the recipient of a fellowship from the Alfred P. Sloan Foundation, a Robert H. Ebert Clinical Scholar Award from the Esther A. and Joseph Klingenstein Fund, an EJLB Foundation award, and a Sidney Kimmel Foundation Award. S.B. is supported by grants from the Canadian Institutes of Health Research and Alberta Heritage for Medical Research.

Supporting Online Material

www.sciencemag.org/cgi/content/full/1093712/DC1 Materials and Methods

Figs. S1 to S4

References

17 November 2003; accepted 22 December 2003 Published online 8 January 2004;

10.1126/science. 1093712

Include this information when citing this paper.

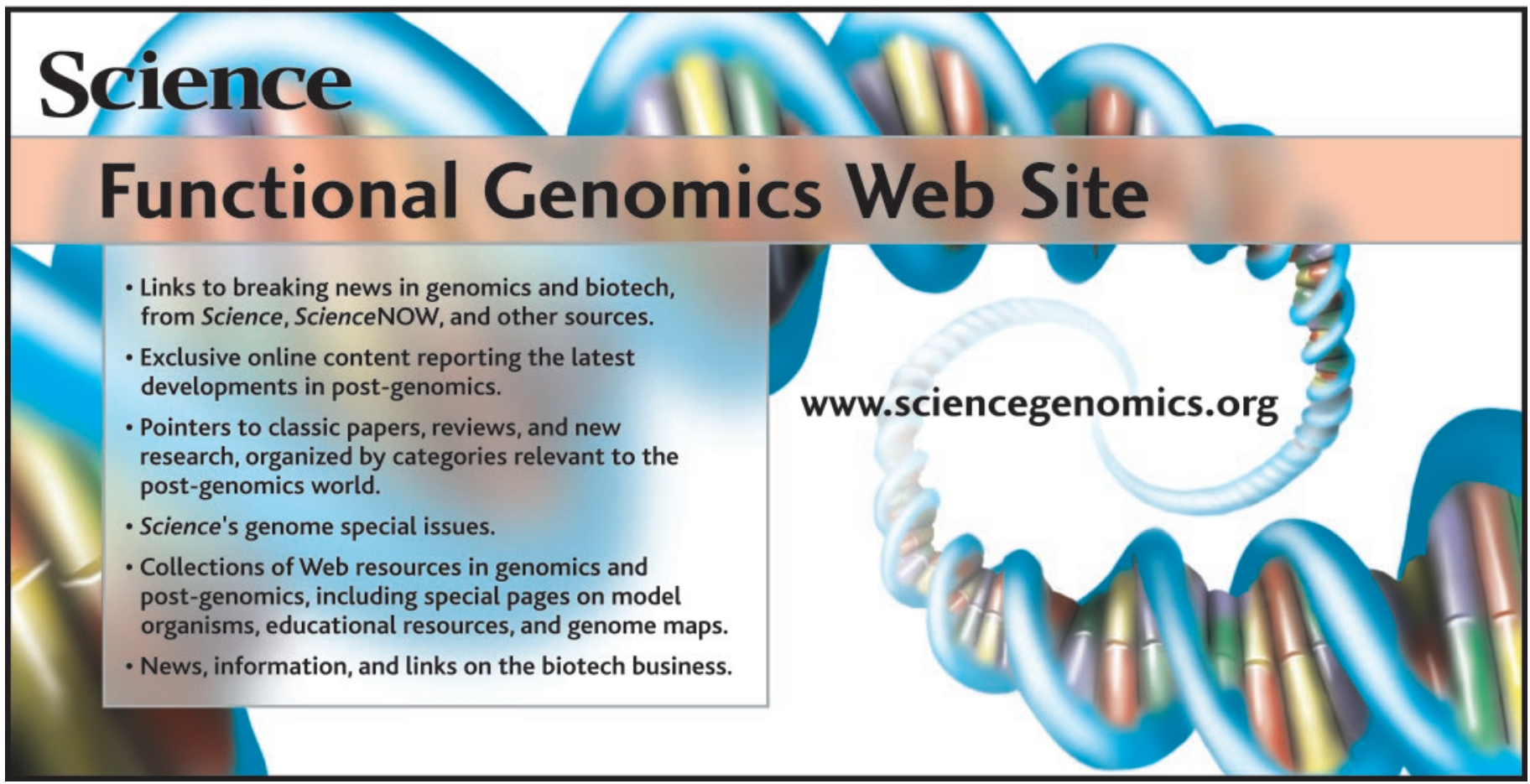

